\title{
Non-linear density dependence in time series is not evidence of non-logistic growth
}

\author{
C. Patrick Doncaster
}

School of Biological Sciences, University of Southampton, Bassett Crescent East, Southampton SO16 7PX, UK

Tel: +442380594352

Fax: +442380594459

Email: cpd@soton.ac.uk

Running headline: Non-linear density dependence in time series 


\begin{abstract}
Time series of population density are often used to seek deviations from logistic regulation by testing for a non-linear decline in per capita growth rate with density. Here I show that this method fails when the interval between observations is not matched by the timing of density impacts on growth. Time series overestimate instantaneous density impacts at low density and underestimate them at high density. More generally, logistic growth produces a deterministically decelerating decline in per capita growth with density if the interval between measures of population size exceeds any lag in density response. Deceleration arises independently out of stochastic density fluctuations, and under-compensating regulation. These multiple influences lead to the conclusion that sequential density estimates provide insufficient information on their own to reveal the identity of non-logistic growth processes. They can yield estimates of density compensation, however, which may suggest time lags in density dependence. Analysis of an empirical time series illustrates the issues.
\end{abstract}

Key-words: Beverton-Holt, Pearl-Verhulst, population regulation, theta-logistic, theta-Ricker.

\title{
1. Introduction
}

The logistic equation provides a simple null model of density dependent population growth, against which to define the processes that regulate population size. Per capita growth rate declines linearly with population density and is therefore characterized by fewer parameters than are required for a curvilinear decline, making it in this sense the simplest density-dependent model (Roughgarden, 1979). Deviations from logistic density dependence have frequently been 
sought from time series of population density by testing for a non-linear decline (e.g., amongst many: Diserud and Engen, 2000; Sæther et al., 2000; Sæther et al., 2002; Sibly et al., 2005).

The approach is attractive because even short time series yield replicate coordinates over time $t$ of density $N_{t}$ and per capita growth $r=\ln \left(N_{t+1} / N_{t}\right)$, with which to model the form of density dependence and strength of density regulation, apparently without need of any life history information. Decelerating or accelerating declines are conventionally interpreted as revealing a weighting of density impacts towards low or high population density. Figure 1 shows an example of a passerine bird with a significantly decelerating decline, from which Sæther et al. (2002) inferred a stronger density regulation at low than at high density. Sibly et al. (2005) noted a prevalence of such decelerating patterns in time series across taxa, and concluded that many animals tend to over-fill their environment, with consequent implications for population management.

In this paper I show that a decelerating decline of $r$ with $N$ is unreliable evidence of nonlogistic growth if based solely on time series data. This is because logistic growth produces a deterministically non-linear decline when $r$ is estimated from interval density measures, unless the measurement interval matches a lag in density impact. Published tests of logistic growth in time series have ignored this potential bias. Moreover, an observed deceleration (or acceleration) can arise from various logistic or non-logistic processes that cannot be distinguished in the absence of more information than is provided by the time series. I will describe the response of $r$ to $N$ for several deterministic models of logistic growth, and variants on logistic growth. These alternatives will then be compared in an evaluation of the Great Tit dataset that also incorporates stochasticity.

[FIG. 1 HERE] 


\section{Models}

\subsection{Logistic growth with instantaneous density impact}

Logistic growth in a population of density $N$ subject to continuous birth and death processes is modeled deterministically in continuous time by the Pearl-Verhulst differential equation:

$r=\frac{d N}{d t} \cdot \frac{1}{N}=r_{0} \cdot\left(1-\frac{N}{K}\right)$

Its defining characteristic is that the instantaneous per capita growth rate $r$ declines linearly with current density $N$, from an intrinsic rate $r_{0}$ in a virgin environment at $t=0$ to zero growth at the population carrying capacity $K$. Its integral with respect to time describes a sigmoidal increase in density from an initial $N_{0}$ as the population grows monotonically over time towards carrying capacity,

measured continuously:

$$
N_{t}=\frac{e^{r_{0} \cdot t} \cdot N_{0}}{1+\left(e^{r_{0} \cdot t}-1\right) \cdot N_{0} / K}
$$

measured at discrete intervals: $\quad N_{t+1}=\frac{e^{r_{0}} \cdot N_{t}}{1+\left(e^{r_{0}}-1\right) \cdot N_{t} / K}$.

The logistic change in density over continuous growth is illustrated in Fig. 2, using values of $r_{0}$ and $K$ consistent with the Fig.- 1 regression on the Great Tit data. The unbroken lines represent growth responses to density changes that are spread over a continuum of time, with an instantaneous response of net recruitment to density (continuous Eq. (2)a). The filled dots on these lines predict the population increments (or decrements) through time from interval measures of continuous growth (recurrence Eq. (2)b).

There is no inherent difference in the underlying ecological mechanism between Eqs (2)ab. Both describe populations that grow monotonically to equilibrium $K$ under an instantaneous 
response to density given by Eq. (1) (Royama, 1992; but cf Eqs (10) and (11) below for applications of Eq. (2)b to metered density responses in discrete growth).

[FIG. 2 HERE]

\subsection{Logistic growth with metered density impact}

Two alternative models can represent logistic dynamics for populations with seasonal recruitment. The discrete-logistic model estimates $r$ from $\Delta N$ given by $N_{t+1}-N_{t}$, expressed per capita. This declines linearly with density in a discrete-time approximation to Eq. (1): $\Delta N / N_{t}=r_{0}\left(1-N_{t} / K\right)$. The model is considered flawed for ecological applications, however, because it predicts crashes to negative density at $N_{t+1}$ from any large $N_{t}>\left(1+r_{0}\right) K / r_{0}$ (Turchin, 2003).

The preferred alternative discrete-time approximation to Eq. (1) for populations with more or less seasonal birth and death processes is the Ricker equation (Ricker, 1954):

$$
r_{t+1}=\ln \left(\frac{N_{t+1}}{N_{t}}\right)=r_{0} \cdot\left(1-\frac{N_{t}}{K}\right)
$$

Its linear decline in per capita growth with density assumes a metered density impact, such that the effect of density on growth is held over for one time unit. This means there is a lag of one time unit either in the growth response to density or the density response to growth (e.g., Brauer and Castillo-Chávez, 2001; Turchin, 2003). For the population of Great Tits, net per capita recruitment of yearlings into the breeding population at time $t+1$ may be assumed to depend on the number of adults at time $t$, for example in response to density-dependent fecundity during the breeding season. Alternatively, writing Eq. (3) as $r_{t}=\ln \left(N_{t+1} / N_{t}\right)=r_{0}\left(1-N_{t} / K\right)$, the same dynamics result from assuming that the population density at time $t+1$ depends on net per capita recruitment into the breeding population at current time $t$, for example in response to the 
availability of unoccupied nest sites at the start of the breeding season. The metered response shows a sigmoidal increase in density over time, at least at low density:

$$
N_{t+1}=N_{t} \cdot \exp \left(r_{0} \cdot\left(1-\frac{N_{t}}{K}\right)\right)
$$

Its dynamics are entirely controlled by $r_{0}$, with monotonic sigmoidal growth for $0<r_{0} \leq 1$ (e.g., Royama, 1992). Larger values push the population through damped oscillations to oscillations between finite limits and on to deterministic chaos (though never to extinction). Figure 2 shows the population overshooting and undershooting the equilibrium $K$ in damped oscillations.

\subsection{Quantifying deviations from logistic growth with $\theta$}

Linear Eq. (1) correctly estimates $r$ as a function of $N$ for a logistic population with instantaneous density regulation of continuous growth (cf incorrect estimate by Eqs (7) below). Deterministic deviations from continuous logistic growth are then conveniently captured by parameter $\theta$ in the generic expansion of Eq. (1) (Gilpin and Ayala, 1973):

$r=\frac{d N}{d t} \cdot \frac{1}{N}=r_{0} \cdot\left(1-\left(\frac{N}{K}\right)^{\theta}\right)$

with $\theta<1$ modeling a decelerating decline in $r$ with $N$, and $\theta>1$ an accelerating decline.

Wherever $r$ is estimated from sequential densities, it will have a relation to $N_{t}$ that depends on the time lag in response to density relative to the time period between consecutive measurements of density. Ricker Eq. (3) applies to time series in which the measurement interval coincides with a lag in the response to $N$. In the event of this coincidence, deterministic deviations from logistic growth are captured by parameter $\theta$ in the generic " $\theta$-Ricker" expansion of Eq. (3) (Thomas et al., 1980): 
$r_{t+1}=\ln \left(\frac{N_{t+1}}{N_{t}}\right)=r_{0} \cdot\left(1-\left(\frac{N_{t}}{K}\right)^{\theta}\right)$,

with $\theta<1$ modeling a decelerating decline in $r$ with $N$, and $\theta>1$ an accelerating decline.

Equation (6) correctly models deviations from logistic growth only if density dependent impacts have a time lag equal to the measurement interval. Yet this model is routinely fitted without any knowledge of density metering, in order to seek evidence of non-logistic growth from $\theta \neq 1$. The accepted wisdom is that $\theta<1$ (decelerating decline of $r$ with $N$ ) indicates density dependence acting most strongly far below carrying capacity whilst $\theta>1$ (accelerating decline) indicates the density response predominating close to $K$.

\section{Results}

\subsection{Deviations from a linear decline in $r$ with $N$ caused by measurement interval}

Time series cannot yield an unbiased estimate of $r$ for populations with instantaneous density regulation, because its value is influenced by the interval between density measures. For example, a perfectly logistic growth in continuous time conforming exactly to Eq. (1) and modeled by $\theta=$ 1 in Eq. (5) will yield the following erroneous estimates of $r$ from consecutive density measures (from Eq. (2)b):

$r_{t}=\ln \left(\frac{N_{t+1}}{N_{t}}\right)=\ln \left(\frac{e^{r_{0}}}{1+\left(e^{r_{0}}-1\right) \cdot N_{t} / K}\right)$.

Here, the per capita growth rate is estimated as a logged ratio (following Sibly et al. 2005 and others), in accordance with the discrete exponential model: $N_{t+1}=R \cdot N_{t}$, where $r=\ln (R)$. One might alternatively consider estimating $r$ from $\Delta N / N$, the per capita change in numbers over the sample interval, in which case a perfectly logistic growth in continuous time conforming to Eq. 
(1) will yield the following erroneous estimate of $r$ from consecutive density measures (also from Eq. (2)b):

$r_{t}=\frac{N_{t+1}-N_{t}}{N_{t}}=\frac{e^{r_{0}}}{1+\left(e^{r_{0}}-1\right) N_{t} / K}-1$.

Clearly, neither Eqs (7)a nor b are compatible with linear Eq. (1). In both cases, their estimates of $r$ have a decelerating response to $N$, modeled by $\theta<1$ in Eq. (5). In effect, the response overestimates the density impact at low densities and underestimates it at densities close to, or above, carrying capacity. Figure 3 contrasts the decelerating response of Eq. (7)a (dashed line) to the linear declines given by both differential Eq. (1) for continuous measurement of instantaneous density regulation and by Ricker Eq. (3) for a metered density response measured at the lag interval. The deceleration inherent to Eqs (7) converges towards linearity if the measurement interval reduces towards the continuous-time measurement of Eq. (1). The seriousness of the bias is illustrated in Fig. 4 by a real example of a yeast culture, which shows almost perfectly logistic growth over continuous time (Fig. 4a). Even measurements taken at 1-hr intervals return a $\theta$ substantially less than unity when modeled by Eq. (6) (Fig. 4b).

\section{[FIGS 3 AND 4 HERE]}

Likewise, for populations with metered density regulation, time series cannot yield a valid estimate of $r$ unless the population is measured at the same lag interval. For example, a logistic population described by Ricker Eq. (3) and modeled by $\theta=1$ in Eq. (6) will yield the following erroneous estimate of $r$ from consecutive density measures taken at intervals of 2 time units (from Eq. (4), substituting $N_{t+1}=f\left(N_{t}\right)$ into $\left.N_{t+2}=f\left(N_{t+1}\right)\right)$ :

$$
r_{t+2}=\ln \left(\frac{N_{t+2}}{N_{t}}\right)=r_{0} \cdot\left(2-\frac{N_{t}}{K} \cdot\left(1+\exp \left(r_{0} \cdot\left(1-N_{t} / K\right)\right)\right)\right) / 2
$$


With the measurement interval twice the lag in density response, Eq. (8) is not compatible with (3) and its estimate of $r$ has a decelerating response to $N$ modeled by $\theta<1$ in Eq. (6). Again, the response overestimates the density impact at low densities and underestimates it at densities close to, or above, carrying capacity. The value of $\theta$ from erroneous application of Eq. (6) will be smaller the more that density impacts on the population between measures, and often the timing of this impact will be an unknown quantity. Exactly the same principle applies if $r$ is measured as $\Delta N / N_{t}$

Any $r$ estimated between census intervals in time series clearly needs to be interpreted with respect to the metering of the density response. This caution has been made by other authors, notably Royama (1992), Turchin (2003), and Gurney and Nisbet (1998), though not in the specific context considered here of testing for evidence of non-logistic growth. The multiplicity of papers claiming to find evidence of non-logistic growth from time series indicate that it has not been understood in this context.

\subsection{Deviations from a linear decline in $r$ with $N$ caused by non-logistic processes}

The conventional explanation for deceleration in the decline of $r$ with $N$ is stronger density impacts at lower density (i.e., density-dependent density dependence). This is only one of several possibilities, however, even if measurement interval is not a contributing factor.

Time series can contain stochasticity in $N_{t}, K$, or $r_{0}$, caused by measurement error or environmental fluctuations (e.g., Wolda, 1989). Deceleration in the decline of $r$ with $N$ arises from any sequence in which $N_{t+1}$ is independent of $N_{t}$. Such a time series will inevitably generate an average linear regression for the response of $r_{t+1}=\ln \left(N_{t+1}\right)-\ln \left(N_{t}\right)$ to the logged density $\ln \left(N_{t}\right)$ with the form: 
$\ln \left(N_{t+1}\right)-\ln \left(N_{t}\right)=-1 \times \ln \left(N_{t}\right)+\operatorname{average}\left\{\ln \left(N_{t+1}\right)\right\}$.

The regression slope of $r_{t+1}$ to $\ln \left(N_{t}\right)$ thus takes a gradient of -1 with intercept at the within-series average $\left\{\ln \left(N_{t+1}\right)\right\}$. The corresponding plot of $r_{t+1}$ against $N_{t}$ therefore has an inherently decelerating decline. It follows that the reported predominance of $\theta<1$ in analyses of time series (e.g., Sibly et al. 2005) may be an illusory pattern, consequent only upon regressing variables against themselves (Doncaster, 2006; see also Nee et al. 2005).

Deceleration in the decline of $r$ with $N$ can also be induced by density undercompensation. Time series provide evidence of density under-compensation from the negative slope of $r_{t+1}$ regressed against $\ln \left(N_{t}\right)$. This response informs on the strength of density dependence, because the time-series estimate of $r_{t+1}$ is itself a linear function of the logged density: $r_{t+1}=$ $\ln \left(N_{t+1}\right)-\ln \left(N_{t}\right)$. A regression slope $\gamma$ with magnitude $|\gamma|=1$ defines perfectly compensating density dependence, with average $N_{t+1}$ remaining constant regardless of $N_{t}$. Density overcompensation is revealed by $|\gamma|>1$, with $N_{t+1}$ overshooting the return from $N_{t}$ towards the population mean. Conversely, density under-compensation is revealed by $|\gamma|<1$, with $N_{t+1}$ undershooting the return (detailed in Doncaster, 2006, following Varley and Gradwell, 1970 and Lande et al., 2002). The Great Tit time series in Fig. 1 has $\gamma=-0.60$, suggesting density undercompensation.

Although Eq. (7)a modeled a biased estimate of $r$ in continuous growth subject to instantaneous density dependence, its unlogged expression is the Beverton-Holt difference equation for metered density dependence (Beverton and Holt, 1957):

$R_{t+1}=\frac{N_{t+1}}{N_{t}}=\frac{R_{0}}{1+\left(R_{0}-1\right) \cdot N_{t} / K}$ 
where $R_{0}=e^{r_{0}}$, the net per capita rate of production before density impacts. Eq. (10) models the density dependence in net per capita production $R_{t+1}$ as an inverse proportion to a linear function of $N_{t}$ (e.g., Kot, 2001). It has $|\gamma|$ below unity and decreasing with lower density, which expresses under-compensating density impacts particularly at low density. This contrasts with the metered density impacts in Ricker Eq. (3) which over-compensate as $N$ approaches $K$ if $r_{0}>1$.

The Beverton-Holt application of Eq. (7)a has a predicted $\theta=0.56$ in Fig. 3 (dashed line) and therefore less curvature than the empirical $\theta=0.44$ in Fig. 1. A more strongly decelerating decline of $r$ with $N$ is modeled by modifying the denominator of Eq. (7)a to include a power function, $b$, that controls density compensation for a given $r_{0}$ and $K$ (Maynard Smith and Slatkin, 1973):

$r_{t+1}=\ln \left(\frac{N_{t+1}}{N_{t}}\right)=\ln \left(\frac{e^{r_{0}}}{1+\left(e^{r_{0}}-1\right) \cdot\left(N_{t} / K\right)^{b}}\right)$.

A value of $b=1$ returns Beverton-Holt Eq. (10) (after taking out of logs) with monotonic damping in under-compensating density dependence. Smaller values of $b$ increase the damping in the monotonic return to $K$, with an associated increase in curvature of $r$ against $N$ over that shown in Fig. 3. Conversely, as $b$ increases above unity, density impacts become over-compensating, passing through damped oscillations to stable oscillations, and eventually deterministic chaos. The effect on the dashed line in Fig. 3 is to reduce its deceleration, and force an accelerating decline at high values of $b$.

\subsection{Application to the Great Tit time series}

It was noted above that perfectly logistic growth in discrete time is modeled by $\theta<1$ in $\theta$-Ricker Eq. (6) if the lag in density response is shorter than the measurement interval (e.g., Eq.(8)). For a population with the parameters of the Fig.-1 Great Tit population, however, an instantaneous 
density response in continuous logistic growth yields $\theta=0.56$ (Fig. 3 dashed line). This raises the possibility that the smaller observed $\theta=0.44$ may result from stochasticity, or density undercompensation, or a weighting on density impacts towards low densities, or some combination of these non-logistic processes.

We can use simulations to test the hypothesis that the observed $\theta$ is explained by density under-compensation as opposed to stochasticity alone in either instantaneous or metered density dependence. With this objective, stochasticity was added to the deterministic models by calculating $N_{t+1}$ from an $N_{t}$ that had fluctuated on a normal distribution either side of the $\log _{e}$ of the deterministic value. The normal distribution used the empirical mean \pm standard deviation of $\ln \left(N_{t}\right)=3.46 \pm 0.29$ to simulate $N_{t}$ over the empirical $t=35$ years. A set of 20 time series were created in this way from each of three models: Ricker Eq. (4) for metered density regulation; Beverton-Holt Eq. (10) for density under-compensation; and Maynard Smith and Slatkin Eq. (11) with strength of density under-compensation estimated from iteratively fitted $b$.

Table 1 compares the resulting estimates of $\gamma$ and $\theta$ to those of the empirical time series on Great Tits (Fig. 1). Discrete logistic model I retains its deterministic character of density overcompensation (Fig. 2) despite the stochasticity, and returns fitted $\theta$ close to its deterministic value. Models II and III in the table simulate density under-compensation, with only Model III close to the empirical $|\gamma|$. The density under-compensation reduces $\theta$, but again only the Model-III value matches closely to the empirical estimate. This match is contingent upon holding $r_{0}$ as a fixed input value when fitting $\theta$ by linear regression of $r$ against $N^{\theta}$. If $r_{0}$ is allowed to vary freely, the unconstrained regression intercept results in fitted $\theta$ with much smaller values for all models caused by the stochasticity (e.g., simulations in Doncaster, 2006), but predicts unrealistically high $r_{0}$ (e.g., Getz and Lloyd-Smith, 2006). 
The model of density under-compensation provides a qualitatively different interpretation to the conventional explanation of $\theta<1$, that the density dependence decreases with density (e.g. in Sæther et al., 2002; Sibly et al., 2005). Density compensation can vary completely independently of density-dependent density dependence, as illustrated by $\theta$-Ricker Eq. (6) where $r_{0}$ controls the former and $\theta$ controls the latter. Without information on population growth rates obtained independently of the time series, it is not possible to distinguish whether or not the density under-compensation is density dependent (i.e., occurs principally at high density).

\section{Discussion}

The analysis has demonstrated how time series data will show a decelerating decline of $r$ with $N$ as an expected outcome of several mutually exclusive processes. It arises with continuous logistic growth from erroneous estimation of $r$ under instantaneous density impact (Eqs (7) and Fig. 3), or any impact with a shorter lag than the measurement interval (Eq. (8)); and it is a non-logistic density under-compensating response to a metered density impact (Beverton-Holt version of Eq. (2)b and Eq. (11)), as well as to density-dependent density dependence (Eq. (6), the conventional explanation). It can also arise directly from bounded stochastic variation (Eq. (9)).

The multiplicity of processes that can influence the estimated $\theta$ lead inescapably to the conclusion that it cannot reliably distinguish how individuals interact at different densities, unless $r$ is measured directly from vital rates (e.g., Fowler, 1981), or it is measured from sequential densities on a population with known $r_{0}$ and either known lag in response to density, or known strength of density compensation. The $\theta$-Ricker model is particularly unsuited to metacomparisons of $\theta$ across time series of different species, which can vary in all of these influences.

To reject the null model of logistic growth simply on the basis of an observed predominance across taxa of $\theta<1$ (e.g., Diserud and Engen, 2000; Sibly et al., 2005, 2006) 
cannot be justified without further analyses of the sort described here for the Great Tit data. Although sequential density measures may reveal little about density-dependent density dependence, they are unquestionably useful for quantifying the overall strength and timing of density regulation, averaged across densities. Analyses of time series by maximum likelihood and information theory now have the capacity to evaluate models of competing mechanisms, to identify lags in density dependence, and distinguish stochastic processes from measurement error (e.g., Dennis and Taper, 1994; Brook and Bradshaw, 2006; Dennis et al., 2006).

\section{Acknowledgments}

This work was supported by grant NE/C003705/1 from the UK Natural Environment Research Council. I thank Tim Coulson and two anonymous reviewers for helpful comments and invaluable insights.

\section{References}

Beverton, R.J.H., Holt, S.J., 1957. On the dynamics of exploited fish populations. Fishery Investigations, Series II 19, 1-533.

Brauer, F, Castillo-Chávez, C., 2001. Mathematical Models in Population Biology and Epidemiology. Texts in Applied Mathematics 40. Springer-Verlag, New York.

Brook, B.W., Bradshaw, C.J.A., 2006. Strength of evidence for density dependence in abundance time series of 1198 species. Ecology 87, 1445-1451.

Carlson, T., 1913. Über Geschwindigkeit und Grösse de Hefevermehrung in Würze. Biochem. Ztschr. 57, 313-334.

Dennis, B., Ponciano, J.M., Lele, S.R., Taper, M.L., Staples, D.F., 2006. Estimating density dependence, process noise, and observation error. Ecol. Monog. 76, 323-341. 
Dennis, B., Taper, M.L., 1994. Density-dependence in time-series observations of naturalpopulations - estimation and testing. Ecol. Monog. 64, 205-224.

Diserud, O.H., Engen, S., 2000. A general and dynamic species abundance model, embracing the lognormal and gamma models. Am. Nat. 155, 497-511.

Doncaster, C.P., 2006. Comment on "On the regulation of populations of mammals, birds, fish, and insects" III. Science 311, 1100c.

Fowler, C.W., 1981. Density dependence as related to life history strategy. Ecology 62, 602-610.

Getz, W.M., Lloyd-Smith, J.O., 2006. Comment on “On the regulation of populations of mammals, birds, fish, and insects" I. Science 311, 1100a.

Gilpin, M.E., Ayala, F.J., 1973. Global models of growth and competition. Proc. Nat. Acad. Sci. USA 70, 3590-3593.

Gurney, W.S.C., Nisbet, R.M., 1998. Ecological Dynamics. Oxford University Press, Oxford.

Kluijver, H.N., 1951. The population ecology of the great tit, Parus m. major L. Ardea 39, 1-135.

Kot, M., 2001. Elements of Mathematical Ecology. Cambridge University Press, Cambridge.

Lande, R., Engen, S., Sæther, B.-E., Filli, F., Matthysen, E., Weimerskirch, H., 2002. Estimating density dependence from population time series using demographic theory and life-history data. Am. Nat. 159, 321-337.

Maynard Smith, J., Slatkin, M., 1973. The stability of predator-prey systems. Ecology 54, 384391.

Nee, S., Colegrave, N., West, S.A., Grafen, A., 2005. The illusion of invariant quantities in life histories. Science 309, 1236-1239.

Pearl, R., 1927. The growth of populations. Quart. Rev. Biol. 2, 532-548.

Ricker, W.E., 1954. Stocks and recruitment. J. Fish. Res. Canada 11, 559-623. 
Roughgarden, , J., 1979. Theory of Population Genetics and Evolutionary Ecology: An Introduction. Macmillan, New York.

Royama, T., 1992. Analytical Population Dynamics. Chapman, Hall, London.

Sæther, B.-E., Engen, S., Lande, R., Arcese, P., Smith, J. N. M., 2000. Estimating the time to extinction in an island population of song sparrows. Proc. R. Soc. Lond. B 267, 621-626.

Sæther, B.-E., Engen, S., Matthysen, E., 2002. Demographic characteristics and population dynamical patterns of solitary birds. Science 295, 2070-2073.

Sibly, R.M., Barker, D., Denham, M.C., Hone, J., Pagel, M., 2005. On the regulation of populations of mammals, birds, fish, and insects. Science 309, 607-610.

Sibly, R.M., Barker, D., Denham, M.C., Hone, J., Pagel, M., 2006. Response to comments on "On the regulation of populations of mammals, birds, fish, and insects". Science 311, $1100 d$.

Thomas, W.R., Pomerantz, M.J., Gilpin M.E., 1980. Chaos, asymmetric growth and group selection for dynamical stability. Ecology 61, 1312-1320.

Turchin, P., 2003. Complex Population Dynamics: A Theoretical/Empirical Synthesis. Princeton University Press, Princeton.

Varley, G.C., Gradwell G.R., 1970. Recent advances in insect population dynamics. Ann. Rev. Entomol. 15, 1-24.

Wolda, H., 1989. The equilibrium concept and density dependence tests. What does it all mean? Oecologia 81: 430-432. 
Table 1

Outputs from stochastic simulations of models for logistic and near-logistic growth.

\begin{tabular}{|c|c|c|c|c|c|}
\hline \multirow{2}{*}{ Model } & \multirow{2}{*}{$\begin{array}{l}\text { From } \\
\text { Eq. }\end{array}$} & \multicolumn{2}{|c|}{$\gamma$} & \multicolumn{2}{|c|}{$\theta$} \\
\hline & & mean & $\mathrm{CI}$ & mean & $\mathrm{CI}$ \\
\hline Empirical time-series (Fig. 1) & (6) & -0.60 & 0.29 & 0.44 & 0.14 \\
\hline \multicolumn{6}{|l|}{ Simulations: } \\
\hline I. Discrete logistic, metered regulation & (3) & -1.34 & 0.08 & 1.01 & 0.06 \\
\hline II. Metered density under-compensation, $b=1.00$ & $(10),(11)$ & -0.79 & 0.10 & 0.57 & 0.07 \\
\hline III. Metered density under-compensation, $b=0.66$ & $(11)$ & -0.58 & 0.06 & 0.42 & 0.04 \\
\hline
\end{tabular}

Parameter $\gamma$ is the least-squares linear regression slope of $r$ against $\ln \left(N_{t}\right)$ and it measures density under-compensation $(|\gamma|<1)$ or over-compensation $(|\gamma|>1)$. Parameter $\theta$ models the deceleration $(\theta<1)$ or acceleration $(\theta>1)$ in the decline of $r$ with $N$, obtained by iterative linear regression of $r$ against $N^{\theta}$ with fixed $r_{0}=1.36$. Each pair of means and $95 \%$ confidence intervals for models IIII was obtained from 20 runs of the simulation. 


\section{Figure Legends}

Fig. 1. Empirical relation of per capita population growth to population size given by a 35-year time series on Great Tits Parus major from Ghent, Belgium 1964 to 1998 (in Sæther et al., 2002). The decelerating regression line is $\theta$-Ricker Eq. (6), with $\theta=0.44$ fitted iteratively in linear regressions of $r$ against $N^{\theta}$ (following Sibly et al., 2005), and constrained to pass from intrinsic $r_{0}$ $=1.36$ individuals per individual per year through carrying capacity $K=33$ individuals at $r=0$. Sæther et al. (2002) independently estimated $r_{0}$ from a Leslie matrix of maximum fecundity rate and lowest age-specific mortality rate; the value is commensurate with other pre-density estimates (e.g., Kluijver, 1951). Fitting Eq. (6) without this forcing yields higher $r_{0}$ and lower $\theta$.

Fig. 2. Three models of deterministic logistic growth in population size from above and below a carrying capacity of $K=33$ individuals, with $r_{0}=1.36$ individuals per individual per year (commensurate with the Fig.-1 Great Tit population). Unbroken lines show responses to instantaneous density impacts measured continuously (Eq. (2)a); filled dots joined by dashed lines are interval measures of these instantaneous impacts (Eq. (2)b); open dots joined by dotted lines are metered density impacts measured at the lag interval (Ricker Eq. (4)).

Fig. 3. Logistic declines in per capita growth $r$ with density $N$ (parameters as Fig. 2). Dashed line joining filled dots shows deceleration caused by taking interval measures of instantaneous density impacts on continuous growth (Eq. (7)a from (2)b). Density regulation measured at the point of impact is modeled by the unbroken line for an instantaneous impact measured continuously (Eq. (1)), and the open dots for a metered impact measured at the lag interval (Ricker Eq. (3)). Note 
that the filled dots also represent the Beverton-Holt model of discrete growth with density undercompensation (Eq. (10)).

Fig. 4. Logistic growth of yeast, from time series by Carlson (1913) cited in Pearl (1927). (a) Logistic Eq. (2)a fitted to continuous growth over time, measured at hourly intervals. (b) Per capita growth rate as a function of density and sampling interval, with regression lines given by $\theta$-Ricker Eq. (6) as in Fig. 1. Filled circles show sampling interval $t=1$ hour with $\theta=0.75$ characterizing the unbroken line of best fit; open circles show $t=2$ hours with $\theta=0.68$ characterizing the dashed line; open triangles show $t=3$ hours with $\theta=0.53$ characterizing the dotted line. 
Figure 1

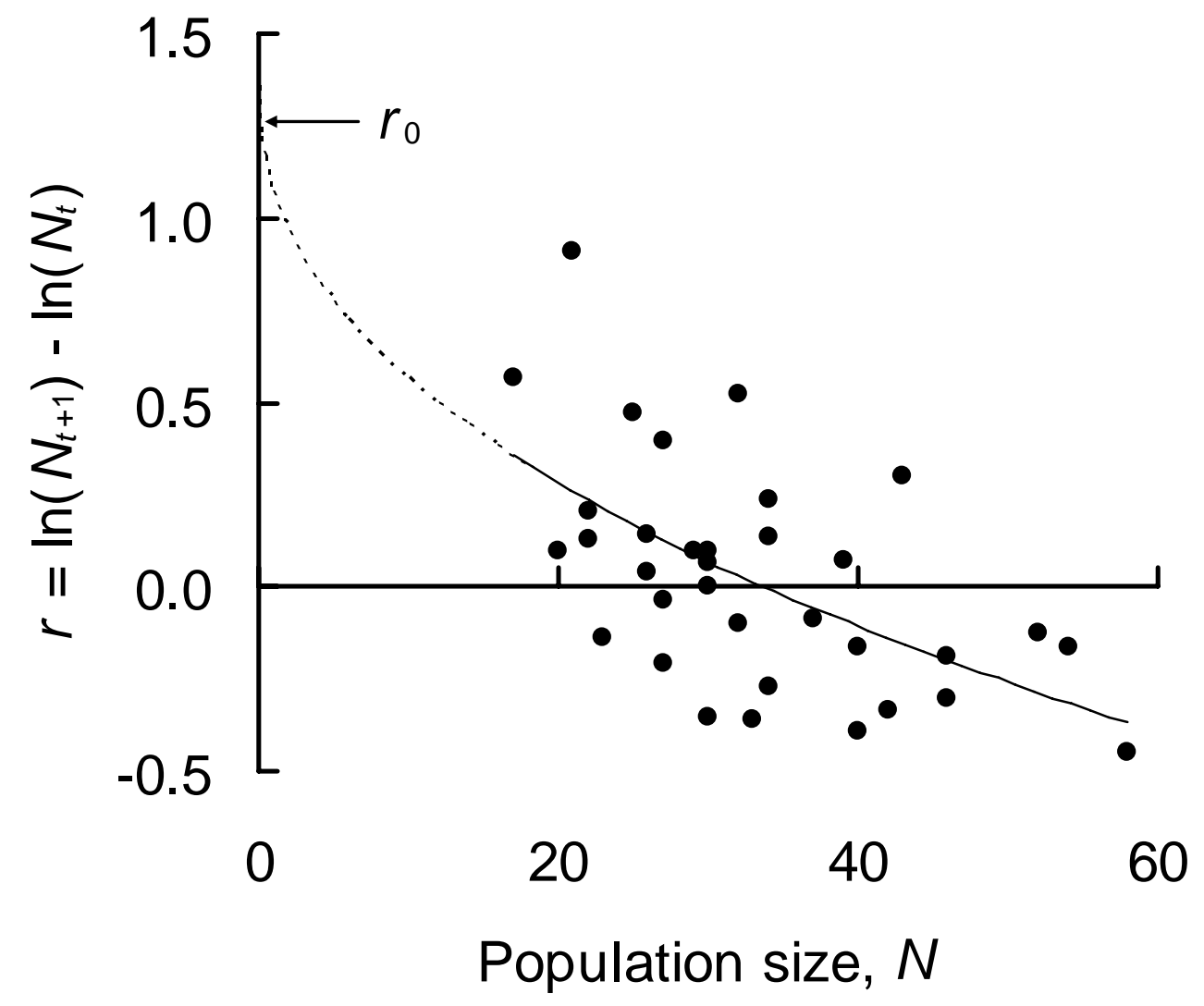


Figure 2

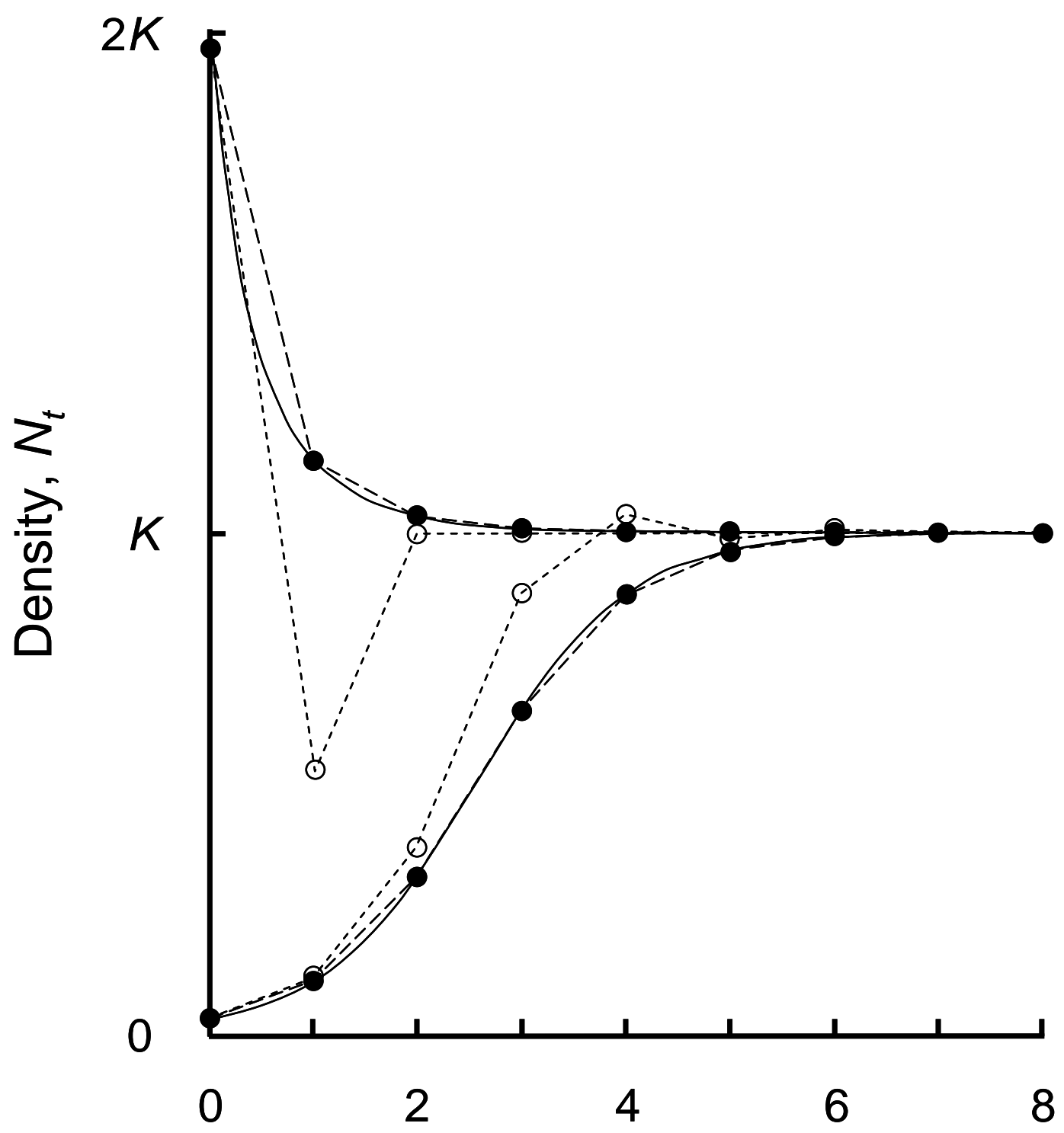

Time, $t$ 
Figure 3

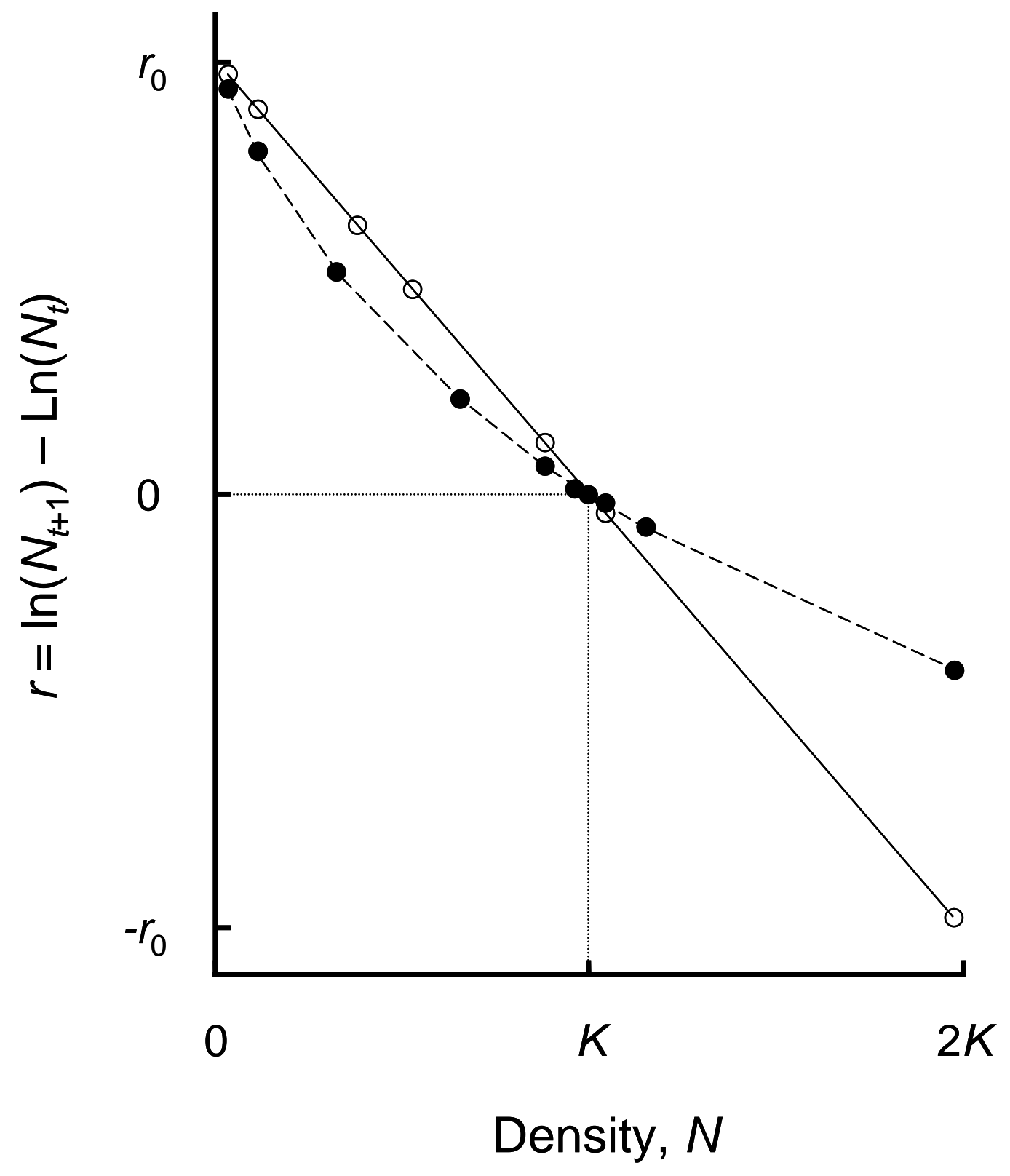


Figure 4(a)

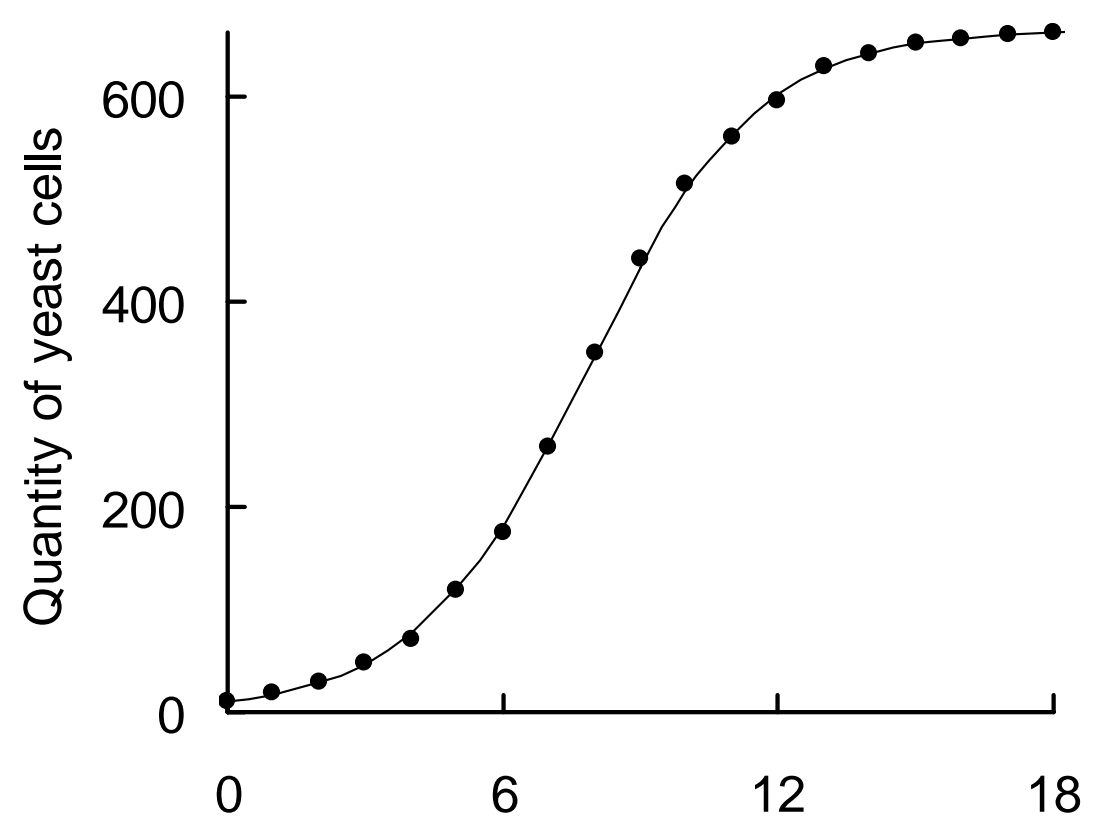

Hours of growth

Figure 4(b)

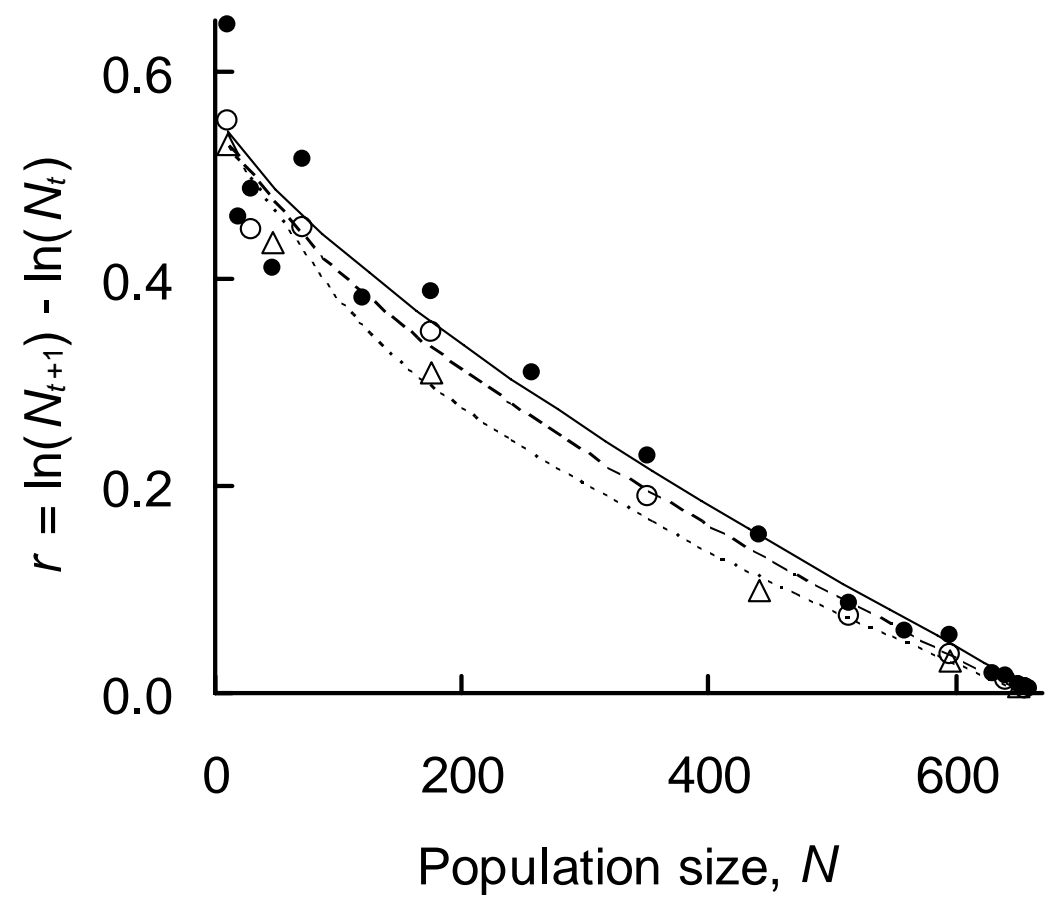

